\title{
$\mathbb{1} \| \varphi^{\prime}$ LEUVEN
}

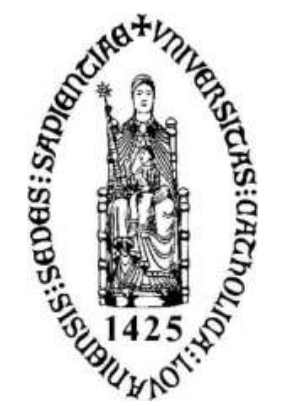

UPC

Z.DRG KU LEUVEN

\section{rTMS in Major Depressive Disorder: Treatment Duration is a Key Element in Treatment Success}

\author{
Deblieck $\mathrm{C}^{1,2}$, Buelens $\mathrm{J}^{1,2}$, Vansteelandt $\mathrm{K}^{1,2}$, Bervoets $\mathrm{C}^{1,2}$ \\ ${ }^{1}$ Department of Psychiatry, UZ Leuven, ${ }^{2}$ University Psychiatric Center, KU Leuven, Belgium
}

\section{Background:}

Since the review by Lefaucheur et al. (2015), rTMS treatment is an alternative treatment option for Major Depressive Disorder (MDD). However, to date no systematic metaanalysis has been published exploring the effect of rTMS.

\section{Objectives:}

1) to examine the effect of rTMS on depression compared to sham.

2) to obtain an optimal estimate of the proportion of patients who showed response under sham versus rTMS treatment.

\section{Materials and Methods:}

A PubMed search was performed, using the identical search terms from the review on rTMS efficacy in MDD as reported by Lefaucheur et al. (2015). The results from the original search were updated until 31-12-2017.

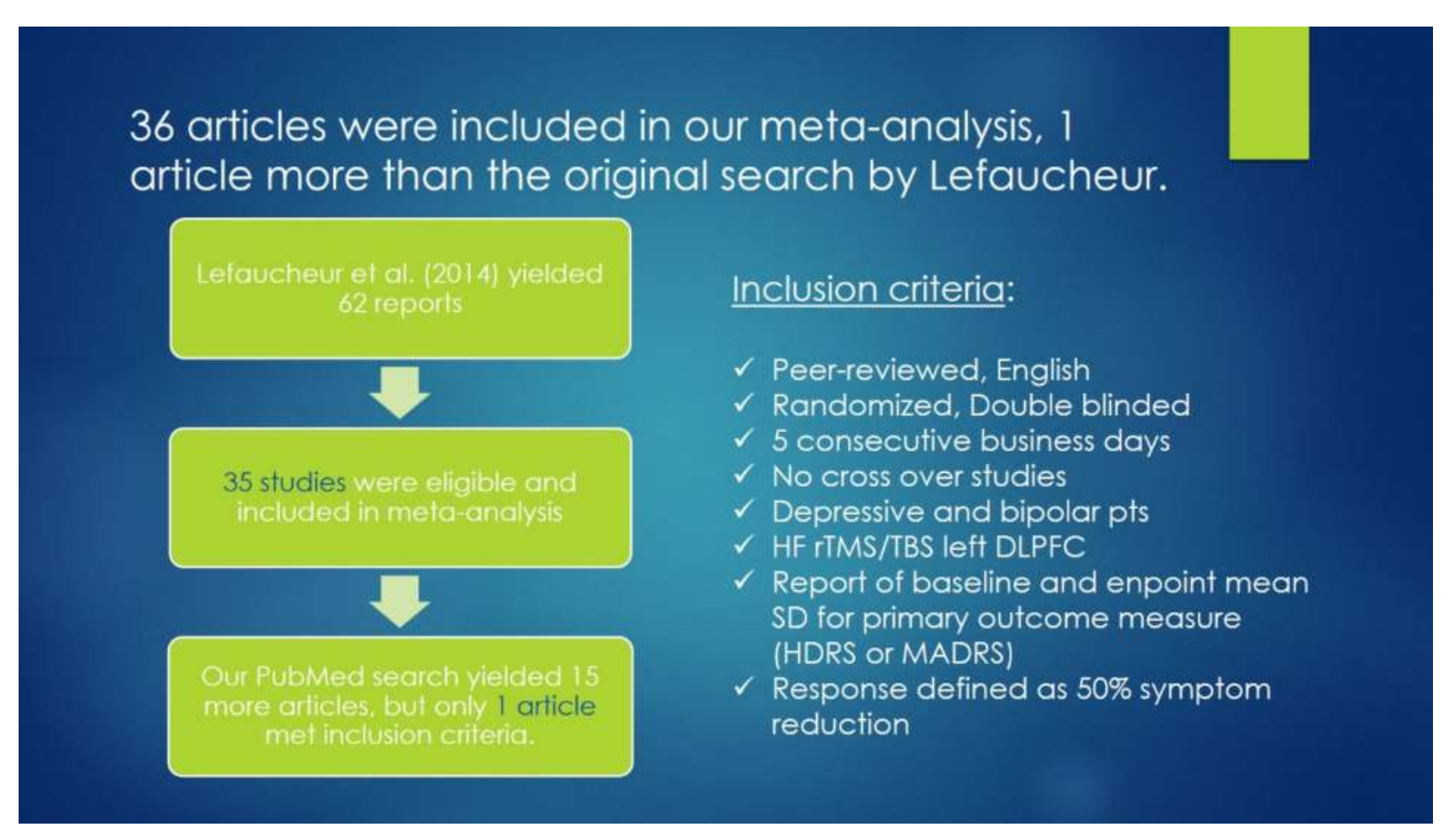

Two Meta-Analyses:

1) to examine the effect of rTMS on depression vs. sham.

2) to obtain an optimal estimate of the proportion of patients who showed response under sham vs. rTMS treatment.

\section{Variables extracted:}

- Duration (Weeks)

- rTMS parameters: f, \# pulses/session, \# of sessions

- Concomitant treatment with Anti-Depressants

- Study population characteristics: TR, in or out patient

- Demographic charecteristics: Age, Gender

\section{Analyses:}

1) to examine the effect of rTMS vs. sham on depression:

- the standardized mean difference and pooled SD was used

- an unbiased, corrected ES was used

2) to obtain an optimal estimate of the proportion of patients who showed response under sham vs; rTMS treatment:

- the Odds Ratio (OR) was used as ES to compare both groups.

Results 1: Effect of TMS compared to sham on depression: The estimated weighted standardized mean difference in the REM is 0.27 (95\% Confidence Interval (Cl): $0.13-0.33)$ implying a small $(0.10)$ to medium effect $(0.30)$ of TMS on depression.
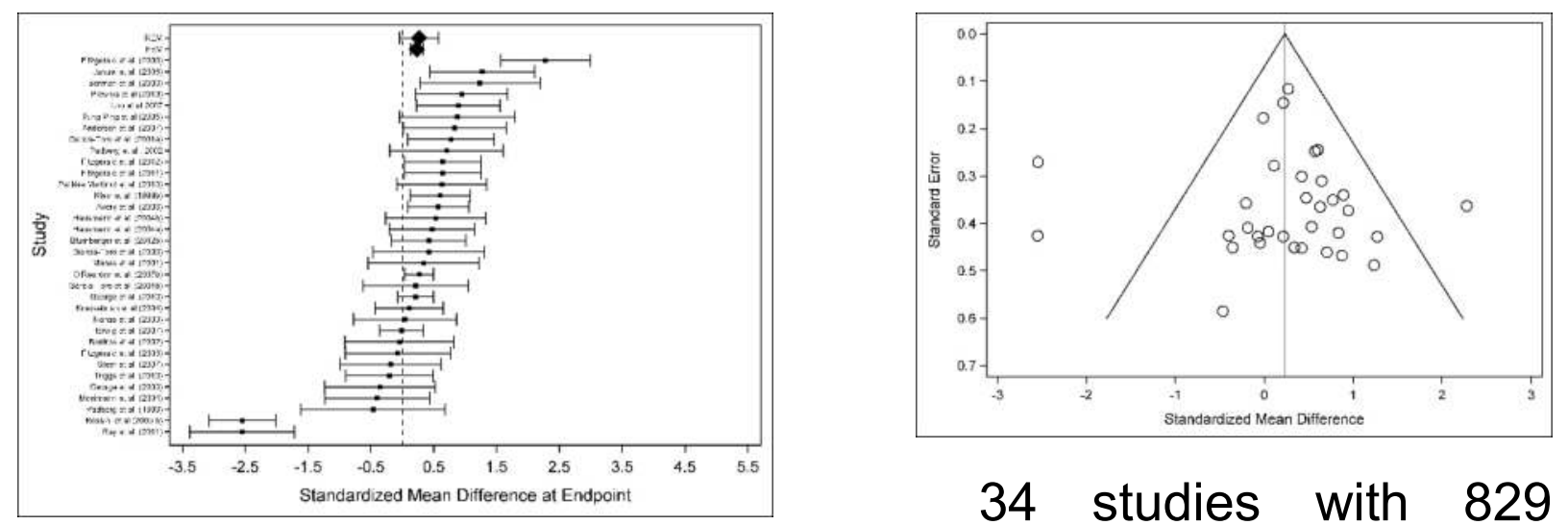

unique patients in the sham-condition and 856 unique patients in the TMS condition.

Results 2: Effect of TMS compared to sham on response: An estimated weighted OR of 2.71 [95\% Cl: 1.71-4.28] was found. Results of this analysis indicates that part of the heterogeneity among studies can be explained by treatment duration, $F(1,15.4)=8.78, p=0.009$, with studies in which patients were treated longer showing a larger ES.
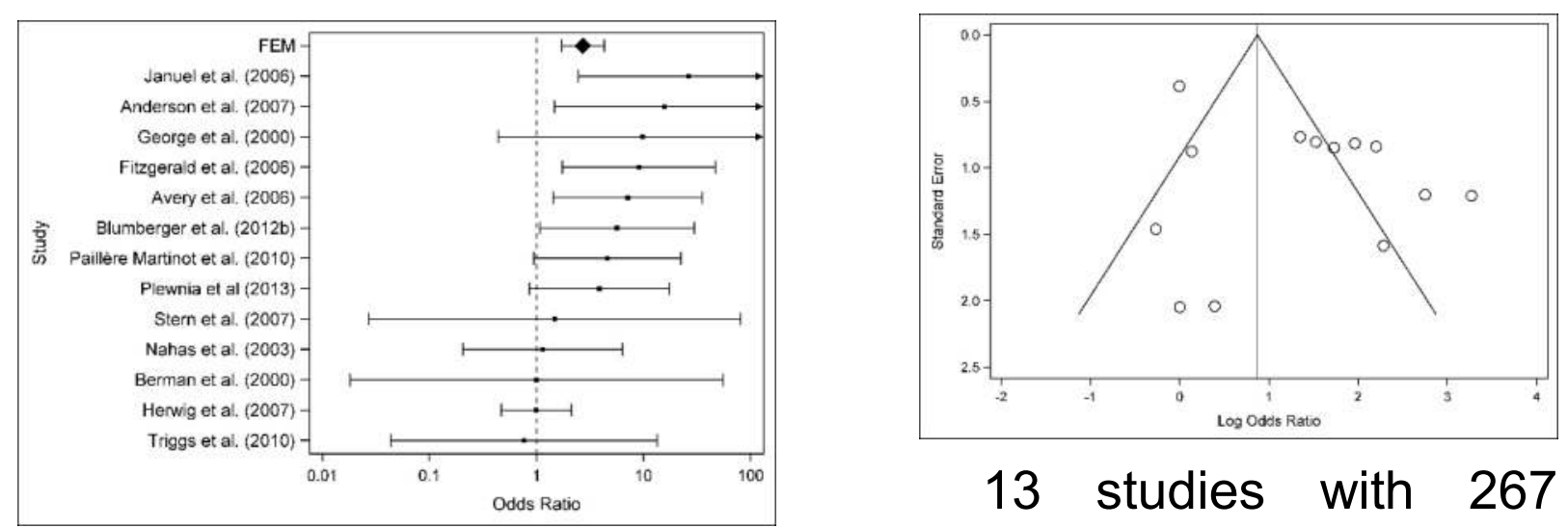

13 studies with 267

unique patients in the sham condition and 318 unique patients in the TMS condition.

Conclusion: Patients with MDD treated with rTMS have a better treatment outcome and a higher chance of remission relative to placebo. Part of the heterogeneity among studies can be explained by treatment duration with studies in which patients were treated longer showing a larger ES 